\title{
Serum High Lipoprotein(a) Levels in Patients with Type 2 Diabetes May Be an Independent Risk Factor for Hypertension
}

\author{
Lingfen Zeng*, Jieming Sun, Ming Cui \\ Department of Internal Medicine, Third People's Hospital, Baiyun District, Guangzhou 510000, Guangdong, China \\ Email: zenglfmail@163.com
}

\begin{abstract}
Objective — To investigate whether serum lipoprotein(a) [Lp(a)] is an independent risk factor for abnormal blood pressure in patients with type 2 diabetes mellitus. Method - Analyzed data collected from diabetes patients and epidemiological survey from January 1,2020 to May 01,2021, with hypertension as a dependent variable, metabolic index such as glycosylated hemoglobin, serum total cholesterol, serum triglyceride and Lp(a) were independent variables, established logistic regression equation, analyze the influence of their variables on dependent variables. Results - The OR value of $\mathrm{Lp}$ (a) is $1.020,95 \%$ confidence intervals $(1.006,1.035), \mathrm{p} 0.006$; The OR value of age is $1.073,95 \%$ confidence interval $(1.028,1.119)$; and OR of the remaining parameters were tested no statistically different, $\mathrm{p}>0.05$. Conclusions The abnormal elevated $\mathrm{Lp}$ (a) level in the serum of type 2 diabetic patients may be related to the occurrence of hypertension. For patients with high Lp(a), Monitoring blood pressure may help to better detect and diagnose hypertension. At the same time, it is suggested that reducing serum Lp(a) level may reduce the risk of hypertension.
\end{abstract}

Keywords: diabetes, lipoprotein(a), hypertension

Up to $60 \%{ }^{[1]}$ of patients with type 2 diabetes are complicated with hypertension, and the specific mechanism remains unclear, which may be related to insulin resistance, atherosclerosis and other factors ${ }^{[2]}$. Current studies ${ }^{[3-5]}$ indicates that lipoprotein (a) [Lp(a)] level serves as an independent risk factor for atherosclerosis, and high levels of Lp(a) are significantly associated with coronary atherosclerotic heart disease and stroke. However, several studies ${ }^{[6-8]}$ displayed that serum Lp(a) level was negatively correlated with insulin resistance, and high serum Lp(a) level could fight insulin resistance and reduce the risk of diabetes. Lp(a) enjoys the dual effect of anti-insulin resistance and increasing atherosclerosis, so does it increase the risk factors for hypertension or reduce the favorable factors for hypertension in patients with type 2 diabetes? Therefore, we intend to analyze the data collected from diabetes patients to explore the relationship between them.

\section{Research objects and methods}

\subsection{Subjects}

Patient data admitted to our hospital from January 1, 2020 to May 1, 2021 and the epidemiological investigation in our region. Patients with severe organ dysfunction and acute infection were excluded, including diabetic ketoacidosis and severe heart failure, patients with New York Grade IV, and patients with renal failure and severe abnormal liver function were excluded. The patient information was derived from the inpatient system, and the patients numbered with the odd number were the objects of this study. In the epidemiological survey of diabetes in this region, random sampling was adopted, and subjects were selected based on residents' wishes. Residents with severe mental disorders were excluded, and patients who met WHO1999 diabetes diagnostic criteria were included in this data analysis. The data of patients complicated with severe infection and other organ dysfunction were excluded. The patients were divided into diabetes mellitus combined with hypertension group and diabetes mellitus without hypertension group.

\subsection{Test methods}

Serum total cholesterol, serum total triglyceride, low density lipoprotein cholesterol and high density lipoprotein cholesterol were determined by enzymatic method; Lp(a) was tested by immunoturbidimetry. All the above items were tested in AU680 automatic biochemical analyzer. HbA1c was tested by high performance liquid chromatography.

\subsection{Statistical methods}

SPSS19.0 software was used for data analysis.Measurement data were expressed as mean \pm standard deviation, and independent $\mathrm{t}$ test was used for comparison between groups. Counting data were counted by Chi-square test. A binary logistic regression equation was established to calculate the OR value and $95 \%$ confidence interval of all factors, by using 
hematological metabolic indexes, age, smoking and hypertension as independent variables and diabetes mellitus with hypertension as dependent variables.

\section{Results}

Table 1. Basic information of patients

\begin{tabular}{cccc}
\hline & $\begin{array}{c}\text { Diabetes mellitus with } \\
\text { hypertension }\end{array}$ & $\begin{array}{c}\text { Diabetes mellitus without } \\
\text { hypertension }\end{array}$ & 63 \\
Cases & 86 & $39 / 24$ & 0.02 \\
Gender (male/female) & $37 / 49$ & $61.0 \pm 10.5$ & 0.01 \\
Age & $66.3 \pm 8.9$ & $5.3 \pm 4.9$ & 0.26 \\
Course of disease (year) & $7.1 \pm 4.2$ & $5.12 \pm 1.35$ & 0.40 \\
CHOL (mmol/L) & $5.33 \pm 1.62$ & $3.02 \pm 3.50$ & 0.69 \\
TG (mmol/L) & $2.80 \pm 2.18$ & $1.55 \pm 1.06$ & 0.85 \\
HDL-C (mmol/L) & $1.50 \pm 0.47$ & $2.54 \pm 0.91$ & 0.08 \\
LDL-C (mmol/L) & $2.51 \pm 0.92$ & $19.8 \pm 24.9$ & 0.79 \\
Lipoproteina (mg/d) & $33.3 \pm 34.0$ & $10.9 \pm 3.1$ & $78 \pm 10$ \\
Glycosylated hemoglobin (\%) & $10.7 \pm 3.4$ & $127 \pm 15$ & $<0.01$ \\
Diastolic blood pressure (mmHg) & $87 \pm 18$ & $157 \pm 32$ & $<01$ \\
\hline
\end{tabular}

Note: CHOL: Total serum cholesterol; TG: serum total triglyceride; HDL-C: High density lipoprotein cholesterol; LDL-C: Low-density lipoprotein cholesterol; $1 \mathrm{mmHg} \approx 0.133 \mathrm{kpa}$.

Table 2. The OR value of each variable of the logistic regression equation

\begin{tabular}{cccc}
\hline & OR & $95 \%$ confidence interval & P \\
\hline Age & 1.073 & $(1.028,1.119)$ & 0.001 \\
Lp(a) & 1.020 & $(1.006,1.035)$ & 0.006 \\
Glycosylated hemoglobin & 0.929 & $(0.929,0.827)$ & 0.209 \\
CHOL & 1.504 & $(0.939,2.412)$ & 0.090 \\
TG & 0.905 & $(0.760,1.079))$ & 0.268 \\
HDL-C & 0.752 & $(0.429,1.317)$ & 0.318 \\
LDL-C & 0.694 & $(0.347,1.389)$ & 0.302 \\
Course of disease & 1.074 & $(0.992,1.163)$ & 0.080 \\
\hline
\end{tabular}

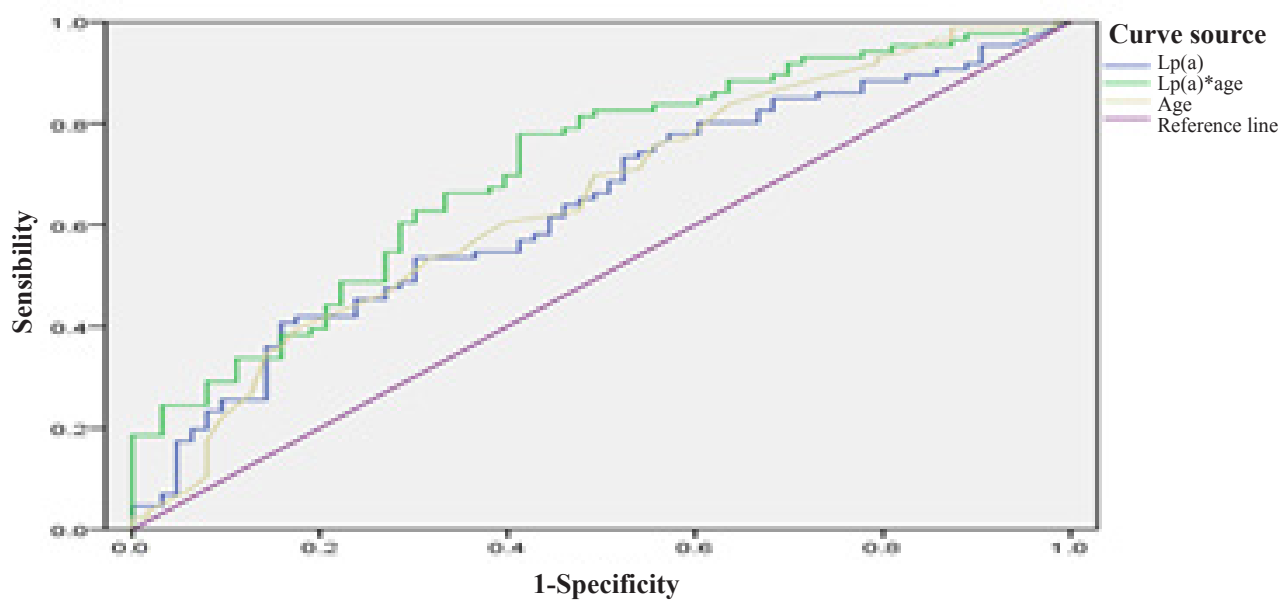

Figure 1. ROC curve of $\operatorname{Lp}(\mathrm{a})$ and age in predicting hypertension 
Table 3. Parametric test of ROC curve

\begin{tabular}{cccc}
\hline & Areas under the ROC curve & $95 \%$ confidence interval & $\mathrm{P}$ \\
\hline Age & 0.649 & $(0.560,0.738)$ & 0.002 \\
Lp(a) & 0.635 & $(0.545,0.724)$ & 0.005 \\
Lp(a)*age & 0.710 & $(0.627,0.793)$ & $<0.001$ \\
\hline
\end{tabular}

\section{Discussion}

$\mathrm{Lp}$ (a) is a cholesterol-rich LDL-like particle that contains Apo(a) in addition to apolipoprotein APOB $100^{[9]}$. Apo (a) is mainly synthesized by the liver ${ }^{[10]}$ and encoded by the LPA gene located in 6q27 staining, while the concentration of serum LPa is determined by the LPA gene ${ }^{[11]}$. The physiological function of $\mathrm{Lp}(\mathrm{a})$ stil remains unclear. Some people believe that $\mathrm{Lp}$ (a) can act as a link between cholesterol transport and fibrinolysis system ${ }^{[12]}$. There are also reports that $\mathrm{Lp}(\mathrm{a})$ is involved in wound healing and tissue repair ${ }^{[13]}$, and some studies have shown that $\mathrm{Lp}(\mathrm{a})$ may act as a carrier of oxidized phospholipids ${ }^{[14]}$. It is still controversial as for whether $\mathrm{Lp}(\mathrm{a})$ is an acute phase protein ${ }^{[15]},{ }^{[16]}$. The pathological effect of $\mathrm{Lp}(\mathrm{a})$ is pertinent to the proatherosclerosis and thrombosis of the vascular system. Serum Lp(a) level is mainly related to genetic factors, and the serum $\mathrm{Lp}$ (a) level is hardly affected by food and other factors ${ }^{[17]}$. It is therefore possible that high $\mathrm{Lp}(\mathrm{a})$ levels may precede hypertension. The prevalence of diabetes combined with hypertension is about $60 \%$, which is $57.7 \%$ in this study, and it is similar to that reported in literature ${ }^{[1]}$. The pathogenesis of hypertension is complex, involving heredity, environment and humoral endocrine, among which insulin resistance is considered to be the common pathophysiological basis of diabetes and hypertension ${ }^{[18]}$. Insulin resistance is associated with $\operatorname{Lp}(\mathrm{a})^{[6-8]}$. Does abnormally high serum $\operatorname{Lp}(\mathrm{a})$ level affect the development of hypertension? As can be seen from the results of our analysis in this study, the Lp(a) level of patients with type 2 diabetes mellitus complicated with hypertension was significantly higher than that of patients without hypertension. Logistic regression was used to further analyze the relationship between various common metabolic factors and the occurrence of hypertension. It was found that serum $\mathrm{Lp}$ (a) level and age had statistically significant effects on the occurrence of hypertension, while HbAlc, cholesterol and triglyceride had no effects on the occurrence of hypertension. Advanced age has been identified as an important factor in the occurrence of hypertension ${ }^{[18]}$, which is consistent with the current understanding. In light of $\mathrm{Lp}(\mathrm{a})$, it is discovered in our study that for elderly patients, every increase of $1 \mathrm{mg} / \mathrm{L}$ was associated with an approximately $2 \%$ increase in the risk of hypertension. As can be seen from the ROC curve, it is also meaningful for $\mathrm{Lp}$ (a) combined with age to predict hypertension, which may be related to arteriosclerosis caused by $\mathrm{Lp}(\mathrm{a})$. This is an issue worthy of our attention. Although there is currently no effective drug targeting high serum Lp(a) levels, relevant studies are being conducted with efforts in this regard with findings that lowering serum Lp(a) can reduce the occurrence of atherosclerosis ${ }^{[19]}$. The significance of this finding lies in that attention should be paid to blood pressure monitoring for patients with high Lp(a), and abnormal blood pressure should be detected and treated in time. The limitation of this study lies in the small sample size, which does not rule out the possibility of statistical bias. Therefore, larger sample size and multi-center studies are needed to further confirm the current conclusions.

\section{Acknowledgments}

This article is supported by Medical and Health Technology Plan Project of Baiyun District, Guangzhou (2020-YL037).

\section{References}

[1] Zhang YQ, Li Y, Dong YG, et al. A nationwide assessment of blood pressure control and the associated factors in Chinese type 2 diabetes mellitus patients. J Clin Hypertens (Greenwich), 2019; 21(11): 1654-1663.

[2] Mancusi C, Izzo R, di Gioia G, et al. Insulin Resistance the Hinge Between Hypertension and Type 2 Diabetes. High Blood Press Cardiovasc Prev. 2020; 27(6): 515-526.

[3] Paré G, Çaku A, McQueen M, el al. Lipoprotein(a) Levels and the Risk of Myocardial Infarction Among 7 Ethnic Groups. Circulation. 2019; 139(12): 1472-1482.

[4] Cho JH, Lee DY, Lee ES, el al. Increased risk of coronary artery calcification progression in subjects with high baseline Lp(a) levels: The Kangbuk Samsung Health Study. Int J Cardiol. 2016; 222: 233-237.

[5] Langsted A, Nordestgaard BG, Kamstrup PR. Elevated Lipoprotein(a) and Risk of Ischemic Stroke. J Am Coll Cardiol. 2019; 74(1): 54-66. 
[6] Paige E, Masconi KL, Tsimikas S, et al. Lipoprotein(a) and incident type-2 diabetes: results from the prospective Bruneck study and a meta-analysis of published literature. Cardiovasc Diabetol. 2017; 16(1): 38.

[7] Rhee EJ, Cho JH, Lee DY, et al. Insulin resistance contributes more to the increased risk for diabetes development in subjects with low lipoprotein(a) level than insulin secretion. PLoS One. 2017; 12(5): e0177500.

[8] Boronat M, Saavedra P, Pérez-Martín N, et al. High levels of lipoprotein(a) are associated with a lower prevalence of diabetes with advancing age: results of a cross-sectional epidemiological survey in Gran Canaria, Spain. Cardiovasc Diabetol. 2012; 11: 81 .

[9] Utermann G. The mysteries of lipoprotein(a). Science. 1989; 246(4932): 904-910.

[10] Kraft HG, Menzel HJ, Hoppichler F, et al. Changes of genetic apolipoprotein phenotypes caused by liver transplantation. Implications for apolipoprotein synthesis. J Clin Invest. 1989; 83(1): 137-142.

[11] Boerwinkle E, Leffert CC, Lin J, et al. Apolipoprotein(a) gene accounts for greater than $90 \%$ of the variation in plasma lipoprotein(a) concentrations. J Clin Invest. 1992; 90(1): 52-60.

[12] Miles LA, Plow EF. Lp(a): an interloper into the fibrinolytic system? Thromb Haemost. 1990; 63(3): 331-335.

[13] Brown MS, Goldstein JL. Plasma lipoproteins: teaching old dogmas new tricks. Nature. 1987; 330(6144): $113-114$.

[14] Bergmark C, Dewan A, Orsoni A, et al. A novel function of lipoprotein [a] as a preferential carrier of oxidized phospholipids in human plasma. J Lipid Res. 2008; 49(10): 2230-2239.

[15] Noma A, Abe A, Maeda S, et al. Lp(a): an acute-phase reactant? Chem Phys Lipids. 1994; 67-68: 411-417.

[16] Mooser V, Berger MM, Tappy L, Cayeux C, Marcovina SM, Darioli R, Nicod P, Chioléro R. Major reduction in plasma Lp(a) levels during sepsis and burns. Arterioscler Thromb Vasc Biol. 2000; 20(4): 1137-1142.

[17] Ferretti G, Bacchetti T, Johnston TP, et al. Lipoprotein(a): A missing culprit in the management of athero-thrombosis? J Cell Physiol. 2018; 233(4): 2966-2981.

[18] Lacruz ME, Kluttig A, Hartwig S, et al. Prevalence and Incidence of Hypertension in the General Adult Population: Results of the CARLA-Cohort Study. Medicine. 2015; 94(22): e952.

[19] Stein EA, Raal F. Future Directions to Establish Lipoprotein(a) as a Treatment for Atherosclerotic Cardiovascular Disease. Cardiovasc Drugs Ther. 2016; 30(1): 101-108. 